\title{
Functional characterisation of the CRE/TATA box unit of type 2 deiodinase gene promoter in a human choriocarcinoma cell line
}

\author{
Gianluca Canettieri ${ }^{1,3}$, Antonella Franchi ${ }^{1,2}$, Rosanna Sibilla1, Ernesto Guzmán ${ }^{3}$ \\ and Marco Centanni ${ }^{1,2}$

\footnotetext{
'Laboratory of Molecular and Endocrine Physiopathology, Department of Experimental Medicine and Pathology, University 'La Sapienza' of Rome, Rome, Italy

'Endocrine Unit, Department of Experimental Medicine and Pathology, University 'La Sapienza' of Rome, Latina, Italy
} \\ ${ }^{3}$ Salk Institute for Biological Studies, La Jolla, California, USA
}

(Requests for offprints should be addressed to Marco Centanni or Gianluca Canettieri; Email: marco.centanni@uniroma1.it or canettieri@salk.edu)

\begin{abstract}
The regulation of expression of type II deiodinase (D2) is a critical mechanism to maintain appropriate intracellular concentrations of tri-iodothyronine in selected tissues. One of the major regulators of D2 concentrations is cAMP, which potently increases human type II deiodinase ( $h D 2$ ) gene transcription in some tissues via a conserved cAMP response element (CRE) located in the promoter region. In addition, the regulatory region of the $h D 2$ gene contains several TATA box/transcription start site (TSS) units, suggesting the presence of different transcripts that might be characterised by different biological properties. However, it is still unclear whether one ore more TATA box/TSS units are needed in response to cAMP or to other signals able to modulate $h D 2$ transcription. In this study we have analysed the ability of cAMP to regulate $h D 2$ in JEG3 cells, a human choriocarcinoma cell line highly responsive to cAMP. Transient transfection assays of different $h D 2$ gene promoter constructs revealed that CAMP induces transcription starting from the most $5^{\prime}$ TSS, located about 80 nucleotides from the CRE. RT-PCR studies have revealed that CAMP activates the expression of a long-lived transcript in JEG3 cells. Site-directed mutagenesis and deletion analysis of promoter constructs have shown that a single CRE/TATA box/TSS unit is needed to confer responsiveness to cAMP. By using chromatin immunoprecipitation studies, we have also demonstrated that the response to CAMP involves the binding of transcription factor CRE binding protein (CREB) to the CRE located in the $h D 2$ promoter. In summary, in JEG3 cells cAMP induces transcription of a long-lived hD2 RNA via CREB and a single CRE/TATA box/TSS unit. This study provides new insights to the regulation of expression of $h D 2$ in placenta.
\end{abstract}

Journal of Molecular Endocrinology (2004) 33, 51-58

\section{Introduction}

Type II deiodinase (D2) is a selenoenzyme that catalyses the monodeiodination of thyroxine $\left(\mathrm{T}_{4}\right)$ or of reverse tri-iodothyronine $\left(\mathrm{T}_{3}\right)$. Its expression and activity appear to be more relevant in some tissues, such as brain, brown adipose tissue, muscle, heart and placenta. In these tissues, in which the maintenance of appropriate concentrations of $\mathrm{T}_{3}$ is critical, D2 seems to play a major part by readily changing the intracellular production of $\mathrm{T}_{3}$ from $\mathrm{T}_{4}$. For this reason, the expression of $\mathrm{D} 2$ needs to be regulated by mechanisms able to increase or decrease the protein concentrations promptly, in response to cellular needs. Evidence has been reported showing that cAMP is one of the major regulators of D2 expression in human, mouse and rat tissues (Bianco et al. 2002). The effect of cAMP seems to be cell-type specific, as cAMP agonists have only a modest effect on $D 2$ gene transcription in pituitary tumour cells (Kim et al. 1998). In addition to cAMP, human type II deiodinase (hD2) RNA levels are regulated by other signals and factors, including $\mathrm{T}_{3}$, dexamethasone (Kim et al. 
1998), the protein kinase $\mathrm{C}$ activator TPA (Imai et al. 2001), and the thyroid transcription factor 1 (Gereben et al. 2001). We and others (Bartha et al. 2000, Canettieri et al. 2000) have described the molecular basis of the effect of cAMP on $h D 2$ gene transcription, showing that its $5^{\prime}$ flanking region contains a functional cAMP response element (CRE), which accounts for the significant transcriptional response to cAMP in human embryonic kidney and in thyroid cells. In addition to the CRE, the $5^{\prime}$ flanking region of the hD2 gene contains several transcription start sites (TSSs) and a $300 \mathrm{nt}$ sequence that can be either expressed or spliced out (Bartha et al. 2000, Canettieri et al. 2000). Using RNA from human placenta, we identified two major TSSs associated with a putative atypical TATA motif (TTTAAAA). Bartha et al. (2000) identified different TSSs in human thyroid located either upstream or downstream of those in placenta, and a canonical TATA box (TATAAA) located $26 \mathrm{nt}$ from the most 5' TSS. The existence of several TATA box/TSS units suggests the presence of different $h D 2$ transcripts that might be characterised by different biological properties. However, it remains unclear whether one or more TATA box/TSS units are needed in response to cAMP or to different signals, able to modulate $h D 2$ transcription.

In this study we have analysed the mechanism by which cAMP regulates hD2 mRNA levels in JEG3 cells, a placenta-derived cell line highly responsive to cAMP treatment.

\section{Materials and methods}

\section{Plasmid construction and site-directed mutagenesis}

The full-length $h D 2-l u c(-1274), h D 2-l u c(-1270)$ mut, hD2-luc ( -560$)$, pSVOALD5' (named in this paper WT, $\triangle \mathrm{CRE}, \mathrm{M} 4$ and empty, respectively) and Zeo A-CRE binding protein (CREB) plasmids have been described previously (Canettieri et al. 2000). The M3 construct was obtained by PCR, using as template the full-length $h D 2$ promoter WT plasmid, together with forward and reverse primers, which both contained a 5' HindIII adapter sequence. The following primers were used for PGR:

Forward: 5'-CGCAAGCTTCTCGAGAATGG TGGGATGGTA-3'
Reverse: 5'-CGCAAGCTTAGATCTTGCTAT CTGTGTGTGGTGGA-3'.

The resulting PCR products were digested and cloned into the HindIII site of the pSVOALD5' plasmid. To perform site-directed mutagenesis, the QuikChange Site-Directed Mutagenesis Kit (Invitrogen) was used, according to the manufacturer's instructions. WT plasmid was used as a template with the following primers: 5'-CAGGGAATGGA GACAAGGCGTCAG-3' and its complementary primer, to obtain the Ml construct, and 5'CGTTAAAGGACGTGAGAAAAAAAAAAAAC-3' and its complementary construct, to obtain the M2 construct. The correct sequence of these mutant plasmids was confirmed by DNA sequencing.

\section{Cell culture, transient transfections and luciferase assays}

JEG3 cells, a human choriocarcinoma cell line, were cultured in Minimum Essential Medium (MEM) supplemented with 10\% fetal bovine serum and $1 \%$ L-glutamine. The day before transfection 200000 cells/well were seeded in six-well plates and grown overnight. For transfection, cells were incubated overnight in OPTIMEM (Invitrogen) with $0.5 \mu \mathrm{g}$ luciferase reporter plasmid, $0.5 \mu \mathrm{g}$ Zeo A-CREB expression vector where indicated and $5 \mu \mathrm{l}$ lipofectamine/well (Invitrogen). Plasmid CMV $\beta$-gal (100 ng/well), which expresses $\beta$-galactosidase under the control of the CMV promoter, was used in all transfections to normalise the luciferase activity. The empty expression vector, Rc/RSV (Invitrogen), was used to maintain a total of $1 \mu \mathrm{g}$ plasmid DNA. The day after transfection, cells were incubated for $4 \mathrm{~h}$ in DMEM containing $0 \cdot 1 \%$ BSA and $10 \mu \mathrm{M}$ forskolin or control dimethylsulphoxide (DMSO) vehicle. After transfection, the cells were lysed in $0.5 \%$ Triton $\mathrm{X}-100,0.25 \mathrm{M}$ Tris (pH 8), and luciferase activity was measured in a Packard luminometer. Results are expressed as luciferase: $\beta$-galactosidase activity ratio, absolute or relative to the empty promoterless vector, and represent the mean \pm S.D. of three independent experiments, each performed in triplicate.

\section{RNA isolation, RT-PCR and Northern blot}

JEG3 cells were grown in $100 \mathrm{~mm}$ dishes until they reached $70-80 \%$ confluence, and $10 \mu \mathrm{M}$ forskolin 
was added for the times indicated. Total RNA was isolated with RNeasy mini Kit (Qiagen) according to the manufacturer's instructions. Reverse transcription was performed with $1 \mu \mathrm{g}$ total RNA using Superscript II (Invitrogen) and random hexamers. The sequence of the primers used for PCR (shown in Fig. 2B) was the following:

A: 5'-GGAGAGAGAGGGACTTTGGAC-3'

B: 5'-GCAATTCAAGAAAGAAACAGGC-3'.

Primers $\mathrm{C}$ and D were as described elsewhere (LP5' and LP3') (Celi et al. 1998). For $\beta$-actin, the following primers were used:

Forward: 5'-CTACAATGAGCTGGGTGT GG-3'

Reverse: 5'-CGGTGAGGATCTTCATGAGG$3^{\prime}$.

Quantitative PCR was performed as described elsewhere (Mayr et al. 2001), using primers specific for hD2 (primers $\mathrm{C}$ and D) and glyceraldehyde-3phosphate dehydrogenase (GAPDH). Northern blot was performed as described previously (Canettieri et al. 2000) using $20 \mu \mathrm{g}$ total RNA from control and forskolin-treated JEG3 cells.

\section{Chromatin immunoprecipitation}

Chromatin immunoprecipitation was performed as described previously (Canettieri et al. 2003). Briefly, cells were incubated with $1 \%$ formaldeyde for $20 \mathrm{~min}$ at room temperature to crosslink proteins to DNA. After crosslinking, cells were lysed $(3 \%$ sarkosyl, $5 \mathrm{mM}$ EDTA, $50 \mathrm{mM}$ Tris-HCl, $\mathrm{pH} 8 \cdot 1$, plus protease inhibitors) and sonicated to reduce the genomic DNA into small fragments (400-600 bp). The lysates were pre-cleared with protein $\mathrm{A}$ -agarose and non-specific antibodies for $2 \mathrm{~h}$ at $4{ }^{\circ} \mathrm{C}$. After pre-clearing, the lysates were incubated overnight at $4{ }^{\circ} \mathrm{C}$ with a rabbit polyclonal CREB antiserum (kindly provided by Dr Marc Montminy) or, as a negative control, with normal rabbit IgG (Santa Cruz). At the end of the incubation, the immunocomplexes were washed extensively and eluted, the crosslinking was reversed and the DNA purified, precipitated and resuspended in TrisEDTA.

The primers used to amplify the hD2 GRE promoter region were the following:

Forward: 5'-GGTTTTCTATTGACTGGAAT CCTGGG-3'

Reverse: 5'-GTGCTTCTCTTTCTCGGAGT CTCAC-3'.

\section{Results}

\section{Regulation of the $h D 2$ gene by cAMP via CRE/CREB in JEG3 cells}

The $h D 2$ promoter contains a GRE that imparts a significant response to cAMP agonists in human thyroid (Bartha et al. 2000) and embryonic kidney cells (Canettieri et al. 2000).

Human choriocarcinoma JEG3 cells represent a suitable model with which to study cAMP regulation of the $h D 2$ gene, as its expression is highly induced by cAMP. Indeed, as shown in Fig. $1 \mathrm{~A}$ and B, Northern blot experiments and Taqman quantitative PCR showed that treatment of JEG3 cells with $10 \mu \mathrm{M}$ forskolin induced a robust, 15-fold increase in a $7 \mathrm{~kb}$ transcript, representing the full-length $\mathrm{hD} 2 \mathrm{mRNA}$. In addition, two smaller transcripts were also detected, probably representing truncated isoforms (Salvatore et al. 1996).

To determine whether the $h D 2$ gene is induced via CRE/CREB in JEG3 cells also, we performed transient transfection studies of promoter constructs. Stimulation of cells transfected with the full length $h D 2$ promoter construct (WT) by $10 \mu \mathrm{M}$ forskolin led to a significant, threefold, increase in luciferase activity, but had no effect on cells transfected with a construct harbouring a $4 \mathrm{nt}$ deletion of the CRE site ( $\triangle \mathrm{CRE}$ ) (Fig. 1C). Consistent with the notion that CREB is the main transcription factor involved in the response to cAMP, transfection of the dominant negative peptide A-CREB completely abolished the effect of forskolin on the WT construct (Fig. 1G).

In our previous study on embryonic kidney cells (HEK293), using an electrophoretic mobility shift assay, we demonstrated the ability of the $\mathrm{hD} 2$ GRE to bind CREB in vitro (Canettieri et al. 2000). A more sensitive approach to determine if a transcription factor is actually bound to the DNA in vivo is the chromatin immunoprecipitation assay. In this novel technique, the endogenous DNA/protein complexes are immunoprecipitated with antibodies raised against the transcription factor of interest and, after washings, elutions and purifications, the DNA is analysed by PCR. This procedure therefore allows detection of chromatin-bound proteins in vivo, under physiological conditions (Orlando 2000). We used chromatin immunoprecipitation to examine whether CREB binds the hD2 CRE in JEG3 cells. PGR amplification of a 
(A)

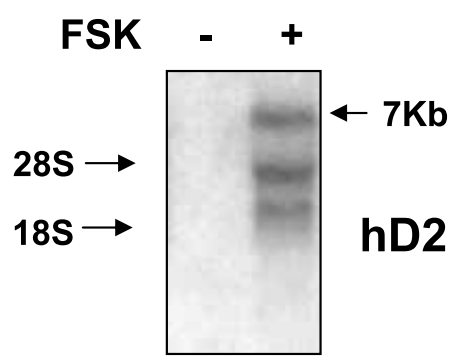

$\beta$-actin

(B)

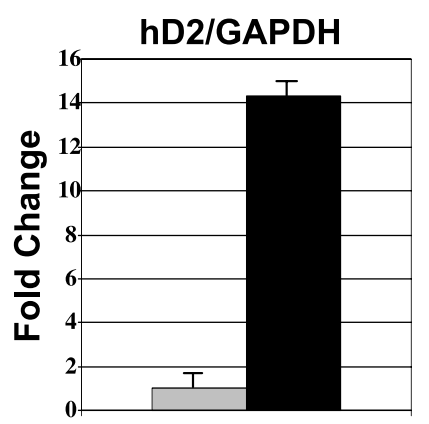

FSK

Figure 1 Activation of transcription of hD2 RNA by cAMP via CRE/CREB in JEG3 cells. (A) Northern blot assay showing hD2 (top) and $\beta$-actin (middle) mRNAs in JEG3 cells treated for $2 \mathrm{~h}$ with $10 \mu \mathrm{M}$ forskolin or DMSO. (B) Fold induction of hD2 RNA relative to GAPDH by Taqman quantitative RT-PCR. (C) Luciferase reporter assay. Cells were transfected overnight with lipofectamine plus $0.5 \mu \mathrm{g}$ luciferase reporter plasmid, $0.5 \mu \mathrm{g}$ of an empty expression vector (EMPTY) or $0.5 \mu \mathrm{g}$ of the expression vector Zeo A-CREB. After transfection, cells were treated for $4 \mathrm{~h}$ with $10 \mu \mathrm{M}$ forskolin or control vehicle DMSO and lysed. Luciferase and $\beta$-galactosidase assays were performed. Results represent the mean \pm S.D. of three independent experiments in triplicate. (D) Chromatin immunoprecipitation. Cells were crosslinked with formaldehyde, lysed and immunoprecipitated with a rabbit polyclonal CREB or normal rabbit antisera for several hours. After immunoprecipitation and extensive washings, the DNA/protein crosslinking was reversed and the DNA extracted. PCR was performed using primers specific for $h D 2$ promoter. FSK, forskolin; RLU, relative light units.

$200 \mathrm{nt}$ region flanking the hD2 CRE was evident only after immunoprecipitation with the specific CREB antiserum, but not with normal rabbit IgG (Fig. 1D), thus demonstrating that CREB binds specifically the hD2 GRE in vivo. No amplification was detected when PCR was performed using primers complementary to the promoter region of the human GAPDH gene (not shown). 

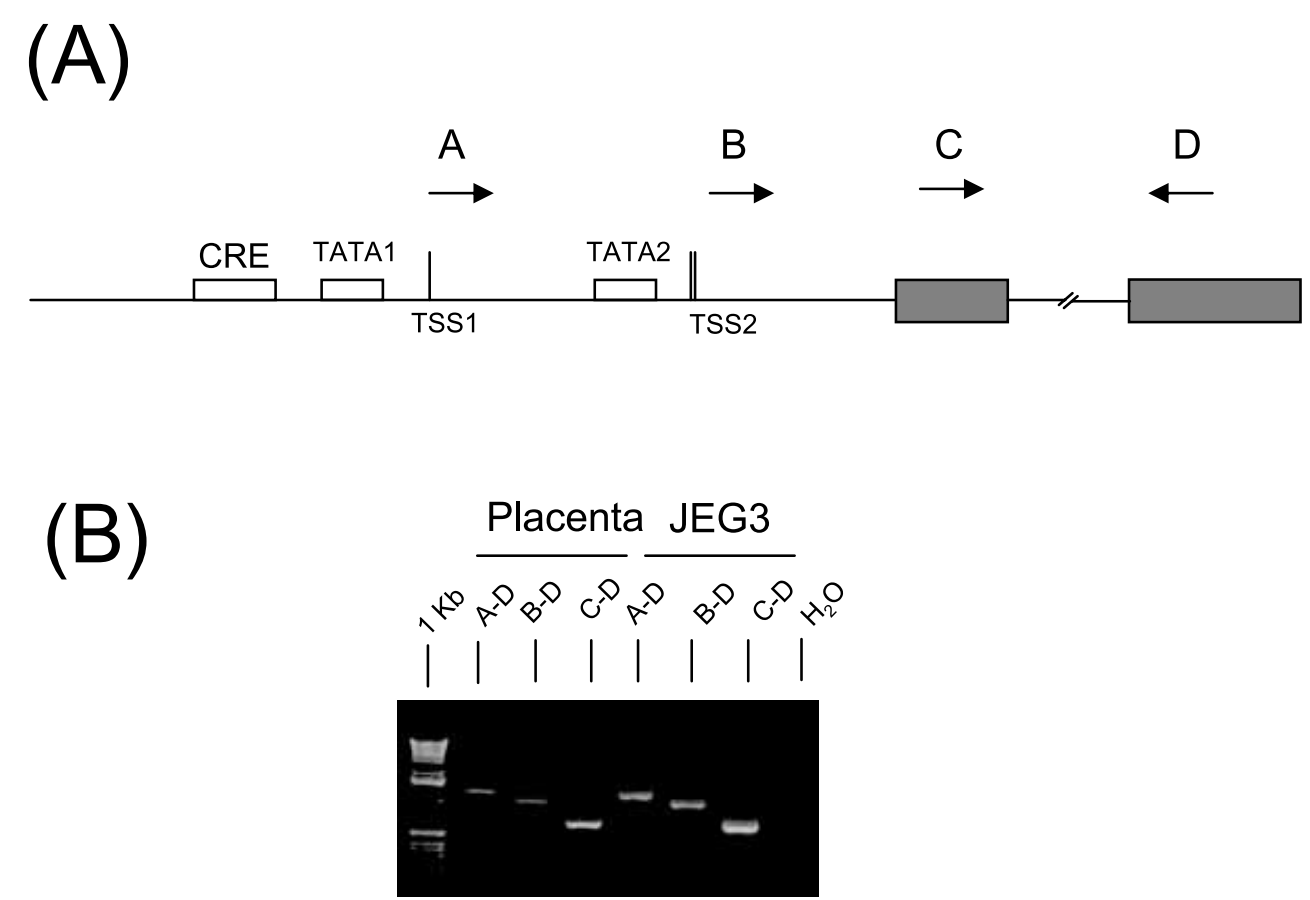

Figure 2 Analysis of the CAMP-induced TSS in JEG3 cells and primary culture of trophoblastic cells. (A) Schematic representation of the $h D 25^{\prime}$ flanking region. The CRE, the TATA boxes and the TSSs are shown. Shaded boxes represent the coding region. Arrows represent the primer used in PCR experiments. (B) RT-PCR showing the TSS in forskolin-treated JEG3 cells and a primary culture of trophoblasts. PCR was performed using primers depicted in (A). JEG3 cells were treated for $2 \mathrm{~h}$ with $10 \mu \mathrm{M}$ forskolin before RNA extraction. $\mathrm{H}_{2} \mathrm{O}$, Negative control without template.

\section{Analysis of the cAMP-induced transcriptional start site}

Several transcriptional start sites have been detected in the $5^{\prime}$ flanking region of the $h D 2$ gene. In particular, in thyroid cells a major TSS (TSS1) has been mapped about 80 nt from the CRE, whereas in a primary culture of human trophoblasts, two major start sites of transcription (TSS2), located about $300 \mathrm{nt}$ from the CRE (Fig. 2A) have been identified.

To analyse the TSS in JEG3 cells upon stimulation with cAMP agonists, we performed RT-PGR studies (Fig. 2B). A clear $1 \cdot 2 \mathrm{~kb}$ band was detectable when a primer starting from the TSS1 was used in forskolin-treated JEG3 cells (lanes A-D). As the expected size of the amplicon in the presence of the $300 \mathrm{nt}$ intron is $1.5 \mathrm{~kb}$, we concluded that cAMP induces a transcript that starts from TSS1 and does not express the $300 \mathrm{nt}$ intron. As expected, the regions starting from the second start site TSS2, in addition to the region encompassing the coding region, were amplified in forskolin-treated JEG3 cells (lanes B-D and C-D). Identical results were obtained by amplifying cDNAs from primary culture of human placenta.

\section{Functional characterisation of the TATA element associated with the CRE}

The hD2 5' flanking region possesses at least two potential CRE-related TATA boxes (Fig. 2A). The first one, named TATA1, is located $26 \mathrm{nt}$ from the TSS1 and the second one (TATA2) is located about 20 bp from the TSS2. To analyse the actual functionality of the two putative TATA elements in response to the cAMP agonist, we performed site-directed mutagenesis, followed by transient transfection analysis (Fig. 3). Mutagenesis of the TATA1, as in the M1 construct, almost completely abolished the response to cAMP. In contrast, the mutagenesis of the TATA2 motif, as in the M2 construct, had no effect on either the basal and/or 


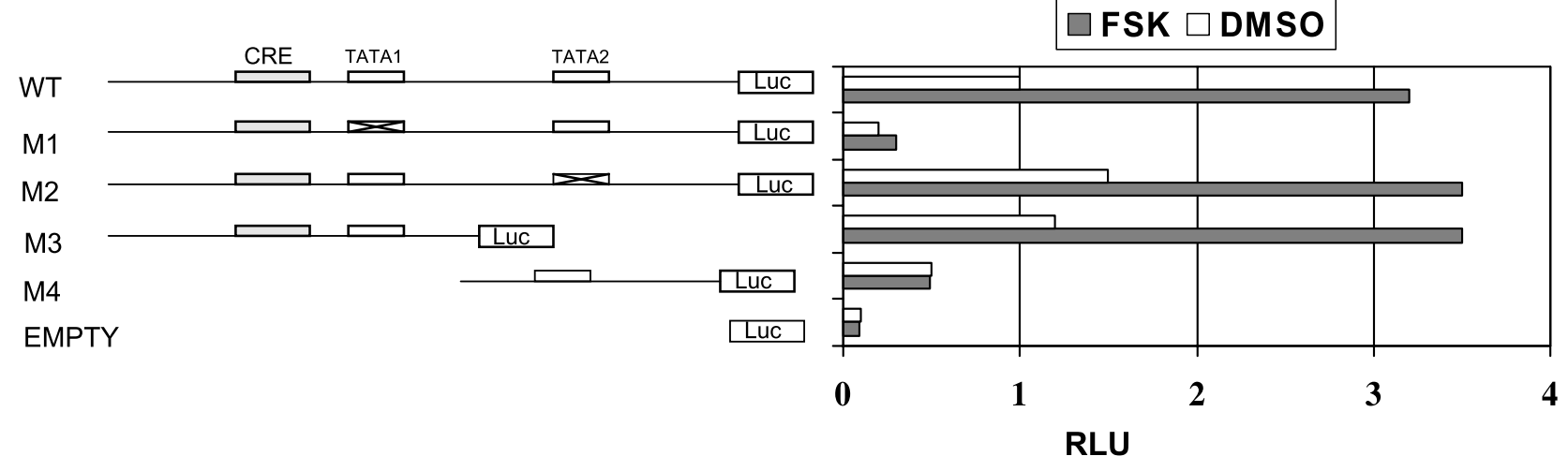

Figure 3 Functional characterisation of the TATA box. Cells were transfected overnight with lipofectamine plus $0.5 \mu \mathrm{g}$ reporter plasmid and $0.5 \mu \mathrm{g}$ of an empty expression vector. After transfection, cells were treated for $4 \mathrm{~h}$ with $10 \mu \mathrm{M}$ forskolin or control vehicle DMSO and lysed. Luciferase and $\beta$-galactosidase assay was performed. Left: Schematic representation of the promoter constructs. Right: Activity of the different constructs. EMPTY, promoterless empty vector.

the cAMP-stimulated promoter construct, indicating that the functional CRE-related TATA box in this cell line is TATA1. To determine whether the region containing only the GRE/TATA1/TSS1 unit is sufficient to direct basal and cAMP-induced transcription of the $h D 2$ gene in JEG3 cells, we transfected a construct from which the region downstream of the TSS1 was deleted (M3). Luciferase activity of either basal and forskolinstimulated cells was comparable to the full-length WT construct, indicating that the complex TSS1/TATAl is fully competent to drive basal and cAMP-induced transcription of the $h D 2$ gene. However, a construct from which the GRE/TSS1/ TATA1 region was deleted (M4) still retained some transcriptional activity compared with the empty vector, suggesting a potential role also for this region in regulating $h D 2$ transcription.

\section{cAMP induces transcription of a long-lived hD2 RNA}

To analyse the stability of the cAMP-induced transcript in JEG3 cells, we performed RT-PGR studies. Consistent with our previous findings, the hD2 RNA was not detectable in untreated cells, but was strongly induced after $2 \mathrm{~h}$ of forskolin treatment (Fig. 4A). The signal remained increased during the following $4 \mathrm{~h}$ and $6 \mathrm{~h}$, and declined progressively after $12 \mathrm{~h}$ and $24 \mathrm{~h}$, suggesting a half-life of about $12 \mathrm{~h}$. To determine whether the amount of transcript detected was related to de novo synthesis of RNA or to post-transcriptional mechanisms, we incubated forskolin-treated JEG3 cells with the RNA polymerase II inhibitor, actinomycin D. Incubation of cells for 3, 6, 12 and $24 \mathrm{~h}$ did not change the expression profile observed
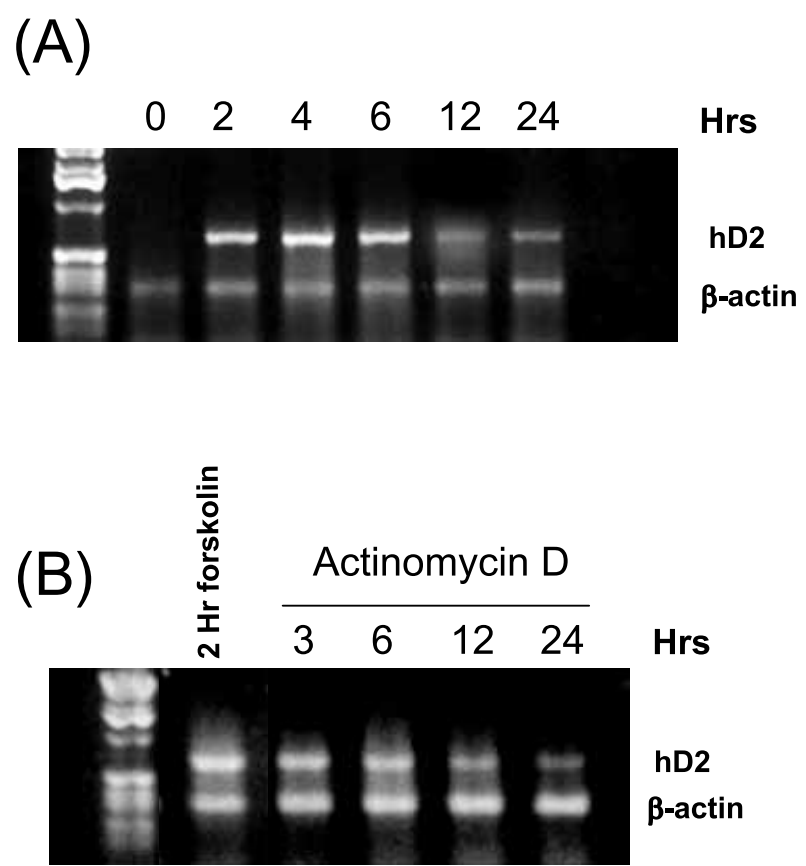

Figure 4 Analysis of hD2 RNA stability. (A) JEG3 cells were treated with $10 \mu \mathrm{M}$ forskolin for the times indicated. (B) Cells were treated with forskolin for $2 \mathrm{~h}$ and then incubated with $5 \mu \mathrm{g} / \mathrm{ml}$ actinomycin $D$ for the times indicated. At the end of the incubation, RNA was extracted from cells and competitive RT-PCR was performed using primers specific for hD2 and human $\beta$-actin. 
in the absence of actinomycin D. This suggests that the levels of RNA detected from $3 \mathrm{~h}$ of cAMP treatment are not related to transcriptional events.

\section{Discussion}

The biological activity of $\mathrm{hD} 2$ in different tissues is tightly regulated by pre- and post-translational mechanisms (Bianco et al. 2002). In this report we have analysed the ability of cAMP to regulate $\mathrm{hD} 2$ mRNA levels in JEG3 cells, a choriocarcinoma cell line highly responsive to cAMP. We have demonstrated that the CRE/TATAl/TSS1 complex is solely responsible for the expression of a long-lived transcript in response to cAMP treatment. We have also shown for the first time that CREB binds the $h D 2$ promoter in vivo, under physiological conditions, and activates transcription in response to cAMP. In our previous report we identified two major transcriptional start sites and, nearby, a putative TATA box (TTTAAAA). This atypical TATA element is frequently found in cAMP-driven promoters (i.e. somatostatin and thyrosine hydroxylase promoters), although the functional significance of these sequences has not been addressed (Montminy 1997). Bartha et al. (2000) found additional TATA element/TSS complexes in the $5^{\prime}$ flanking region. One of these complexes (TATAl/TSS1) is located at less than $100 \mathrm{bp}$ from the CRE. It has previously been reported that a CRE confers its greatest inducibility when located less than $100 \mathrm{bp}$ from the TATA box, being less active at major distances (Montminy 1997). According to this notion, in this report we have demonstrated that the mutagenesis of the most 5' TATA box (TATAl) disrupts both basal and cAMP-stimulated transcription of $h D 2$ promoter constructs and that cAMP induces the expression of a transcript starting from the most 5' TSS. Also, this TSS1/TATA1 unit is solely responsible for the effect of cAMP, as deletion of the region downstream of this core promoter did not affect the cAMP response. In contrast, the mutagenesis of the TATA2 element did not change either basal or cAMP-induced transcription, indicating that this sequence is not functional, at least in this cellular model. It is likely that the presence of a pyrimidine-rich region around the TSS2 may supply the lack of functional TATA box in this core promoter (Roeder 1996). Taken together, these data indicate that a single-unit CRE/TATA box/ TSS1 is necessary and sufficient to impart cAMP inducibility to the $h D 2$ gene.

In addition to transcriptional control, the intracellular concentrations of D2 are regulated at different levels, including mRNA processing and stability and post-translational modifications (Bianco et al. 2002). Both the $5^{\prime}$ and $3^{\prime}$ untranslated regions of $\mathrm{hD} 2$ mRNA seem to have an important role in regulating $\mathrm{D} 2$ concentrations. In particular, the 5' UTR possesses several TSSs, one small intron and three different open reading frames that may affect the efficiency of translation, whereas the 3' UTR has several AU-rich elements (ARE, AUUUA) that could affect RNA stability (Gereben et al. 2002). Our study indicates that cAMP stimulates the expression of a transcript starting from the most $5^{\prime}$ TSS that does not contain the $300 \mathrm{nt}$ intron. Interestingly, the 5' UTR starting from the TSS1 has been shown to decrease the translation efficiency of D2 protein. Therefore, cAMP seems to induce the expression of a transcript that is stable but, perhaps, with a low efficiency of translation. This finding is peculiar, because all the transcripts previously described in thyroid and other tissues (Kim et al. 1998, Bianco et al. 2002) showed a short, $2 \mathrm{~h}$ half-life. A possible explanation for this observation could be that, in addition to the transcriptional control, cAMP regulates hD2 mRNA stabilisation. A similar effect of cAMP on mRNA stability has been demonstrated with several other genes, including GLUT1, GLUT5 and the glucocorticoid receptor (Dong et al. 1989, Cornelius et al. 1991, Gouyon et al. 2003). Interestingly, it has recently been shown (Adams et al. 2003) that cAMP prolongs the half-life of human renin mRNA by stimulating the expression of $\mathrm{HuR}$ and CP1, two proteins that bind AUUA motifs. As hD2 mRNA possesses 11 AUUA elements in its $3^{\prime}$ UTR, it is possible that a similar mechanism may account for the observed prolonged half-life in cAMP-treated JEG3 cells. Different combinations of stability/translational efficiency may thus regulate intracellular D2 concentrations, depending on the stimulus and the cellular context. Further studies are needed to clarify these issues.

In summary, in JEG3 cells cAMP induces $h D 2$ transcription via CREB. The cAMP response is entirely dependent on a single GRE/TATA box/TSS unit that allows transcription of a 
long-lived mRNA. This study provides new insights to understanding of the basal expression of $h D 2$ and its regulation by physiological stimuli.

\section{Acknowledgements}

This work was supported by a grant from the Italian Ministero dell'Università e della Ricerca (Progetti di interesse nazionale, Cap. 8969 2002). GC was supported by a fellowship granted by Istituto Pasteur, Fondazione Cenci-Bolognetti. We thank Dr Marc Montminy for reagents and advice, and Dr Loredana Fiorentino for suggestions and discussion on the manuscript. We also thank Dr Felice Petraglia and Dr Pasquale Florio for RNA from primary cultures of human trophoblast.

This work is dedicated to the memory of Giuditta.

\section{References}

Adams DJ, Beveridge DJ, van der Weyden L, Mangs H, Leedman PJ \& Morris BJ 2003 HADHB, HuR, and CP1 bind to the distal $3^{\prime}$-untranslated region of human renin mRNA and differentially modulate renin expression. Fournal of Biological Chemistry 278 44894-44903.

Bartha T, Kim S, Salvatore D, Gereben B, Tu H, Harney J, Rudas P \& Larsen PR 2000 Characterization of the 5'-flanking and $5^{\prime}$-untranslated regions of the cyclic adenosine $3^{\prime}, 5^{\prime}$-monophosphate-responsive human type 2 iodothyronine deiodinase gene. Endocrinology 141 229-237.

Bianco AC, Salvatore D, Gereben B , Berry M \& Larsen P 2002 Biochemistry, cellular and molecular biology, and physiological roles of the iodothyronine selenodeiodinases. Endocrine Reviewes $\mathbf{2 3}$ 38-89.

Canettieri G, Celi FS, Baccheschi G, Salvatori L, Andreoli M \& Centanni M 2000 Isolation of human type 2 deiodinase gene promoter and characterization of a functional cyclic adenosine monophosphate response element. Endocrinology 141 1804-1813.

Canettieri G, Morante I, Guzman E, Asahara H, Herzig S, Anderson SD, Yates JR \& Montminy M 2003 Attenuation of a phosphorylation-dependent activator by an HDAC-PP1 complex. Nature Structural Biology 10 175-181.
Celi F, Canettieri G, Yarnall D, Burns D, Andreoli M, Shuldiner A \& Centanni M 1998 Genomic characterization of the coding region of the human type II $5^{\prime}$-deiodinase gene. Molecular and Cellular Endocrinology 147 49-52.

Cornelius P, Marlowe M, Call K \& Pekala PH 1991 Regulation of glucose transport as well as glucose transporter and immediate early gene expression in 3T3-L1 preadipocytes by 8-bromo-cAMP. Fournal of Cell Physiology 146 298-308.

Dong Y, Aronsson M, Gustafsson JA \& Okret S 1989 The mechanism of cAMP-induced glucocorticoid receptor expression. Correlation to cellular glucocorticoid response. Foumal of Biological Chemistry 264 13679-13683.

Gereben B, Salvatore D, Harney JW, Tu HM \& Larsen PR 2001 The human, but not rat, dio2 gene is stimulated by thyroid transcription factor-1 (TTF-1). Molecular Endocrinology 15 112-124.

Gereben B, Kollar A, Harney JW \& Larsen PR 2002 The mRNA structure has potent regulatory effects on type 2 iodothyronine deiodinase expression. Molecular Endocrinology 16 1667-1679.

Gouyon F, Onesto C, Dalet V, Pages G, Leturque A \& Brot-Laroche E 2003 Fructose modulates GLUT5 mRNA stability in differentiated Caco-2 cells: role of cAMP-signalling pathway and PABP (polyadenylated-binding protein)-interacting protein (Paip) 2. Biochemical fournal 375 167-174.

Imai Y, Toyoda N, Maeda A, Kadobayashi T, Fangzheng G, Nishikawa M \& Iwasaka T 2001 Type 2 iodothyronine deiodinase expression is upregulated by the protein kinase A-dependent pathway and is downregulated by the protein kinase C-dependent pathway in cultured human thyroid cells. Thyroid 11 899-907.

Kim SW, Harney JW \& Larsen PR 1998 Studies of the hormonal regulation of type $25^{\prime}$-iodothyronine deiodinase messenger ribonucleic acid in pituitary tumor cells using semiquantitative reverse transcription-polymerase chain reaction. Endocrinology 139 4895-4905.

Mayr BM, Canettieri G \& Montminy MR 2001 Distinct effects of cAMP and mitogenic signals on CREB-binding protein recruitment impart specificity to target gene activation via CREB. PNAS 98 10936-10941.

Montminy M 1997 Transcriptional regulation by cyclic AMP. Annual Review of Biochemistry 66 807-822.

Orlando V 2000 Mapping chromosomal proteins in vivo by formaldehyde-crosslinked-chromatin immunoprecipitation. Trends in Biochemical Science 25 99-104.

Roeder RG 1996 The role of general initiation factors in transcription by RNA polymerase II. Trends in Biochemical Science $21327-335$.

Salvatore D, Bartha T, Harney JW \& Larsen PR 1996 Molecular biological and biochemical characterization of the human type 2 selenodeiodinase. Endocrinology 137 3308-3315.

Received in final form 30 January 2004 Accepted 27 February 2004 